\title{
RELACIONES FAMILIARES ENTRE PADRES E HIJOS /AS CON TRASTORNO DE ESPECTRO AUTISTA (TEA) A TRAVÉS DEL JUEGO COMO ACTIVIDAD PRINCIPAL EN LA INTERVENCIÓN DE TERAPIA OCUPACIONAL: ESTUDIO DE CASOS, EN COLEGIOS DE LA COMUNA DE MAIPÚ (RM. CHILE)
}

\author{
FAMILY RELATIONS BETWEEN PARENTS AND CHILDREN WITH ASD THROUGH \\ PLAY, AS MAIN ACTIVITY IN THE OCCUPATIONAL THERAPY INTERVENTION: \\ CASES STUDY, IN SCHOOLS OF THE COMMUNE OF MAIPÚ (RM. CHILE)
}

\section{Blanca Paloma Flores del Villar ${ }^{1}$}

\begin{abstract}
RESUMEN
Las alteraciones del Trastorno de Espectro Autista (TEA) se caracterizan por deficiencias persistentes en la comunicación e interacción social, además de la existencia de patrones restrictivos y repetitivos de comportamiento, intereses o actividades referencia (American Psychiatric Association, p28, 2014). La familia es el primer agente socializador, en ella nacen las bases de las relaciones sociales y de los vínculos afectivos. Este estudio explora y describe las relaciones familiares establecidas dentro del juego espontáneo, en padres, madres e hijo/a con diagnóstico temprano de TEA. Mediante un diseño de casos múltiples, de carácter cualitativo, exploratorio y no experimental, se estableció un plan de intervención de Terapia Ocupacional el cual se aplicó a tres familias con niño/as con TEA. Para documentar las experiencias, se utilizaron: un diario de campo y la videograbación de las intervenciones, y para determinar la percepción de los padres se aplicaron entrevistas semiestructuradas. Con la participación de la familia durante el tratamiento de Terapia Ocupacional a través del juego, los resultados confirman que las relaciones entre familia e hijo/a con TEA son medianamente modificadas y en algunos casos sustantivamente modificadas. Concluyéndose que la relación entre familia e hijo/a con TEA podrían verse modificadas, mediante el juego como ocupación principal, a través de la intervención de Terapia Ocupacional. Observándose además nuevas habilidades de interacción tanto en familias como en niños.
\end{abstract}

PALABRAS CLAVE

Terapia Ocupacional, Relaciones Familiares, Trastorno Autístico, Conducta Infantil.

1 Terapeuta Ocupacional. Diplomatura de terapia ocupacional Escuela de Salud de Universidad de Granada. Convalidación título chileno de terapeuta ocupacional, Universidad de Chile. Máster en terapia ocupacional en infancia Universidad de Castilla-la Mancha. Magister Ocupación y Terapia Ocupacional Universidad de Chile. paloma.flrs@gmail.com 


\begin{abstract}
The changes in Autistic Spectrum Disorder (ASD) are characterized by persistent deficiencies in communication and social interaction, besides of restrictive and repetitive patterns of behavior, interests and activities. The family is the first and foremost social agent, where basis of social relationships and affective bonds are created. This study explores and describes the family relations established through spontaneous plays, in families, parents and children with an early diagnosis of ASD. Through a study of multiple cases, of qualitative nature, exploratory and not experimental, a plan of intervention of Occupational Therapy was established and applied to three families with children who have ASD. In order to keep record of experiences, during interventions a field diary and recorded videos were used, and in order to determine parents' perception, semi-structured interviews were conducted. Results showed that family relations between parents and children with ASD are, at least moderately modified and in some cases substantially modified, due to the participation of the family through plays in the treatment of Occupational Therapy. The conclusion would be that family relation between parents and children with ASD can be modified through the play, as activity and principal occupation which is relevant for the development of the child from the intervention of Occupational Therapy. New interaction skills with families and children were observed.
\end{abstract}

\title{
KEYWORDS
}

Occupational Therapy, Family Relations, Autistic Disorder, Child Behavior

Recibido: 27/11/2019

Aceptado: 01/09/2021

\section{INTRODUCCIÓN}

Tomando en cuenta los datos encontrados sobre el aumento en la prevalencia diagnóstica en base a la experiencia clínica con Trastorno Espectro Autista y familia (Departamento de Discapacidad y Rehabilitación, División de Prevención y Control de enfermedades, Subsecretaría de salud pública, Ministerio de Salud de Chile, p. 13, 2011), este estudio surge la inquietud de generar conocimiento sobre cómo son las relaciones establecidas entre padres e hijos; de qué manera la intervención del Terapeuta Ocupacional desde el juego puede incidir estas relaciones, y los posibles beneficios que supondría para ambos la modificación de estas.

Estudios anteriores mencionan que la instrucción de la familia por parte del Terapeuta Ocupacional para ser coterapeutas en el proceso de tratamiento de su hijo permite extender estas relaciones y las maniobras terapéuticas e indicaciones a su hogary al entorno del niño, lo que favorece a su vez la prolongación en tiempo y contexto de la intervención, y presume una mejoría en la calidad de vida del niño y de los padres (Brezis et al., 2015).
El juego presenta características propias: "surge de la motivación intrínseca y placer del niño, es libre y espontáneo, le permite autoexpresarse, descubrir el ambiente que le rodea, y le proporciona competencias a través de su propia experiencia en el hacer" (Parham \& Fazio, p. 55, 2007).

Así mismo en este estudio donde el juego entendido como área de desempeño ocupacional fundamental en la infancia para el desarrollo de competencias y habilidades en el niño desde la visión del Terapeuta Ocupacional, sería el eje central de interacción entre padres y niño, para: "promover la interacción, favorecer el intercambio de experiencias, y enriquecer el espectro de alternativas a investigar, ampliando las referencias del manejo del entorno del niño" (López, 2014, p. 70) incidiendo el desarrollo de nuevos comportamientos, intereses o actividades.

Así, asumiendo como característica diagnóstica del TEA las deficiencias persistentes en la comunicación e interacción social en diversos contextos, además de la existencia de patrones restrictivos y repetitivos de comportamiento, intereses o actividades (American Psychiatric 
Association, p. 28, 2014) con este estudio se busca llegar a una aproximación de las relaciones establecidas entre familia y niño con TEA mediante las experiencias de juego entre ambos desde el interior de la sala.

Para el investigador es importante dar voz y lugar a la familia en el proceso de intervención de las relaciones establecidas con su hijo con TEA.

Debido a que hay aspectos de las relaciones, como la manera de ser o establecerse de las mismas, las propiedades emergentes de estas, etc. que solo pueden ser observadas desde un punto de vista cualitativo. Y es mediante las experiencias, perspectivas y puntos de vistas de las propias personas que establecen dicha interacción, como pueden ser estudiadas estas relaciones. Para ello en este estudio, se toma a la familia, para sea esta la que por sí misma, de cuenta cómo son las relaciones que establece con su hijo con TEA y cómo mediante la interacción de juego entre familia y niño pueden verse modificadas las relaciones establecidas.

Existen estudios anteriores que observaron aumentos en las habilidades de crianza positiva en las madres tras una terapia de interacción entre madres e hijos con TEA (Agazzi, Tan, Ogg, Armstrong \& Kirby, 2017).

A esta información hay que añadirle los sentimientos observados que los padres presentan con respecto a la crianza de su hijo con TEA: desconfianza de las capacidades parentales (McConachie \& Diggle, 2007; Schultz, Stichter, Herzog, McGhee \& Lierheimer, 2012), y gran estrés (Beaudoin, Sébire \& Couture, 2014), afectando directamente las relaciones que mantienen con su hijo.

En mucho de los estudios existentes (Vernon, Koegel, Dauterman, \& Stolen, 2012; Beaudoin, Sébire, \& Couture, 2014; McKnight, O'Malley-Keighran, \& Carroll, 2016; Zlomke, Jeter, \& Murphy, 2017) se elabora un programa que procura enseñar a los padres cómo interactuar y comunicarse con sus niños pequeños con TEA a través de intervenciones de capacitación de padres y que podrían promover el desarrollo de las habilidades sociocomunicativas de sus hijos. (Beaudoin, Sébire \& Couture, 2014).

\section{MÉTOdo}

Este estudio descriptivo, exploratorio, de casos múltiple, tomando como objeto de estudio la interacción social (relación entre familia e hijo con TEA) y sus cambios mediante el juego, para aportar más información y conocimientos a los insuficientes estudios actuales, surge dicha investigación.

Estableciéndose como objetivos generales, explorar los procesos de interacción entre familia y niño con TEA en las relaciones establecidas dentro del juego entre familia y niño con TEA, y establecer los cambios y modificaciones en la interacción en las relaciones establecidas dentro del juego entre familia y niño con TEA, a partir de la intervención de Terapia Ocupacional basada en el juego.

Es un estudio de carácter cualitativo, ya que para el investigador es importante dar voz y lugar a la familia en el proceso de intervención de las relaciones establecidas con su hijo con TEA; y de tipo longitudinal (Hernández Sampieri, Collado, \& Lucio, 2010), debido a que explora los cambios establecidos a través del tiempo.

Además, se considera de carácter exploratorio y descriptivo (Dankhe G.L., 1976). Ya que, con él, se pretende aumentar el grado de familiaridad con fenómenos relativamente desconocidos, como son las relaciones establecidas en la interacción de familia y niño con TEA dentro del ambiente de juego, con el fin de obtener más información sobre el contexto particular e investigar el comportamiento humano dentro del mismo contexto.

El estudio se estructuro en unas 20 sesiones de inicio a fin de la intervención, de una hora de duración, con una frecuencia de dos veces por semana. Estructuradas en una primera sesión de toma de contacto con la familia presentación del estudio y realización de formulario y entrevista Pre-intervención, seguida de dos sesiones de observación inicial, diecisiete sesiones de intervención y una sesión final de realización de entrevista Post-intervención. 


\begin{tabular}{|c|c|c|c|}
\hline \multicolumn{4}{|c|}{ 1. PROGRAMA DE INTERVENCIÓN } \\
\hline $\begin{array}{l}\text { NÚMERO } \\
\text { DE SESIÓN }\end{array}$ & ACTIVIDAD & OBJETIVO & ASPECTOS A OBSERVAR \\
\hline 0 & $\begin{array}{l}\text { Reunión con los padres, } \\
\text { Observación del niño en } \\
\text { sala. }\end{array}$ & $\begin{array}{l}\text { Realización de formularios, } \\
\text { entrevistas y test. }\end{array}$ & \\
\hline 1 y 2 & $\begin{array}{l}\text { Observación del TO. } \\
\text { Interacción entre familia } \\
\text { y niño. } \\
\text { Búsqueda de motivación } \\
\text { intrínseca de juego en el } \\
\text { niño. }\end{array}$ & $\begin{array}{l}\text { Reconocer las características de } \\
\text { las relaciones establecidas entre } \\
\text { familia y niño en las interaccio- } \\
\text { nes observadas. } \\
\text { Conocer en el niño motivación } \\
\text { y preferencias en tipo u objetos } \\
\text { de juego, así como rutinas y } \\
\text { organización de este. }\end{array}$ & $\begin{array}{l}\text { Motivación intrínseca, espon- } \\
\text { taneidad, disfrute, flexibilidad, } \\
\text { mantenimiento, preferencias en } \\
\text { el tipo de juego, elementos u } \\
\text { objetos de juego, metodología } \\
\text { organización y rutina de juego, } \\
\text { etc. }\end{array}$ \\
\hline 3,4 y 5 & $\begin{array}{l}\text { Búsqueda sensorial } \\
\text { predominante. }\end{array}$ & $\begin{array}{l}\text { Regulación de nivel de alerta y } \\
\text { nivel de actividad. } \\
\text { Mantenimiento de óptimo nivel } \\
\text { de alerta. } \\
\text { Exploración intencional del } \\
\text { entorno. }\end{array}$ & $\begin{array}{l}\text { Preferencias, búsquedas sen- } \\
\text { soriales, reacción a los sonidos, } \\
\text { luces, movimiento, tacto, etc.) }\end{array}$ \\
\hline 6 & $\begin{array}{l}\text { Acompañamiento de } \\
\text { padres al juego del niño. }\end{array}$ & $\begin{array}{l}\text { Proporcionar seguridad, opor- } \\
\text { tunidades, tiempo, libertad de } \\
\text { elección, fomento la interacción } \\
\text { social y el aprendizaje de habi- } \\
\text { lidades y conductas, generaliza- } \\
\text { ción de aprendizajes, apoyo y } \\
\text { adaptación, etc. }\end{array}$ & \\
\hline 7 y 8 & $\begin{array}{l}\text { Incorporación de los } \\
\text { padres al juego. }\end{array}$ & $\begin{array}{l}\text { Introducción en el juego del } \\
\text { niño: aumento de interacción. }\end{array}$ & $\begin{array}{l}\text { En cuanto a las interacciones: la } \\
\text { acepta, la permite, la incorpora, } \\
\text { atiende, comprende, responde } \\
\text { a ellas, etc. }\end{array}$ \\
\hline $\begin{array}{l}9,10,11 \\
\text { y } 12\end{array}$ & $\begin{array}{l}\text { Abrir círculos de } \\
\text { comunicación. }\end{array}$ & $\begin{array}{l}\text { Contacto visual compartido, } \\
\text { indicaciones, gestos, expresión } \\
\text { de emociones y respuesta a } \\
\text { las mismas, contacto, atención } \\
\text { compartida, imitación, lenguaje } \\
\text { intencional, etc. }\end{array}$ & $\begin{array}{l}\text { Reconoce y responde a las } \\
\text { aproximaciones de manera } \\
\text { positiva. } \\
\text { Mantiene la conexión visual o } \\
\text { verbal. Involucra al otro en su } \\
\text { actividad. } \\
\text { Responde a las expresiones y } \\
\text { comienza a desarrollar gestos } \\
\text { para comunicarse. } \\
\text { Muestra interés } \\
\text { Muestra reacciones emociona- } \\
\text { les acordes. }\end{array}$ \\
\hline
\end{tabular}




\begin{tabular}{|c|c|c|c|}
\hline $\begin{array}{l}\text { NÚMERO } \\
\text { DE SESIÓN }\end{array}$ & ACTIVIDAD & OBJETIVO & ASPECTOS A OBSERVAR \\
\hline 13 y 14 & $\begin{array}{l}\text { Consolidación de } \\
\text { aprendizajes }\end{array}$ & $\begin{array}{l}\text { Consolidación y refuerzo de } \\
\text { los objetivos perseguidos } \\
\text { anteriormente }\end{array}$ & $\begin{array}{l}\text { Objetivos conseguidos y no } \\
\text { conseguidos. }\end{array}$ \\
\hline \multirow[t]{2}{*}{15,16 y 17} & \multirow[t]{2}{*}{$\begin{array}{l}\text { Extendiendo círcu- } \\
\text { los de comunicación. } \\
\text { (interacción) }\end{array}$} & Comunicación bidireccional. & $\begin{array}{l}\text { Abre, mantiene y cierra círculos } \\
\text { de comunicación y responde a } \\
\text { las conductas. } \\
\text { Inicia acciones intencionales } \\
\text { con los objetos mientras que } \\
\text { se mantiene enganchado en la } \\
\text { interacción con el otro. } \\
\text { Comparte información. } \\
\text { Responde a los gestos con sus } \\
\text { propios gestos. } \\
\text { Utiliza expresiones, acciones y } \\
\text { sonidos para comunicarse. }\end{array}$ \\
\hline & & Solución de problemas. & $\begin{array}{l}\text { Utiliza secuencias intencionales } \\
\text { y complejas de gestos y pala- } \\
\text { bras para resolver problemas } \\
\text { o satisfacer necesidades de } \\
\text { manera compartida. } \\
\text { Planifica nuevas acciones para } \\
\text { el reto y las comparte. } \\
\text { Explora de manera intencional } \\
\text { para entender cómo funciona } \\
\text { el mundo y encontrar nue- } \\
\text { vos caminos para solucionar } \\
\text { problemas. } \\
\text { Desarrolla un vocabulario de } \\
\text { gestos para comunicar pensa- } \\
\text { mientos y emociones complejas. } \\
\text { Comienza a reconocer patrones } \\
\text { emocionales. }\end{array}$ \\
\hline 18 у 19 & $\begin{array}{l}\text { Intervenciones mediadas } \\
\text { por los padres, donde el } \\
\text { TO quedará en segundo } \\
\text { plano. }\end{array}$ & $\begin{array}{l}\text { Poner en práctica y consolidar } \\
\text { los objetivos aprendidos. }\end{array}$ & \\
\hline 20 & $\begin{array}{l}\text { Reformulación de } \\
\text { entrevistas. }\end{array}$ & $\begin{array}{l}\text { Recoger experiencia y obser- } \\
\text { vaciones de los padres tras la } \\
\text { intervención. }\end{array}$ & \\
\hline
\end{tabular}


Previo al inicio del estudio fue presentado al Comité de Ética de Investigación en Seres Humanos, Facultad de Medicina, Universidad de Chile, el cual revisó la documentación y aprobó su realización

En una primera etapa se exploraron varios establecimientos educacionales, de los cuales, luego de la invitación solo dio respuesta la Escuela Especial Mi Nuevo Arlequín, una escuela particular, subvencionada, situada en la comunidad de Maipú, que atiende a menores con trastornos graves de la comunicación (Trastorno Especifico Lenguaje y Trastorno Espectro Autista). Quien dispuso de una de sus salas equipadas con elementos propios de una sala de intervención de Integración Sensorial: elementos colgantes, piscina de pelotas, materiales de distintas texturas, colchonetas, módulos, encajables, etc. de la que el investigador y Terapeuta Ocupacional con formación en Infancia, Integración Sensorial y modelo DIR-Floortime entre otras compartió uso con las familias y niños durante las sesiones de juego.

Tres casos fueron seleccionados por la directora y coordinadora del colegio en base al cumplimiento de los criterios de inclusión: Familias con niño/as con diagnóstico temprano de TEA y compromiso de asistencia de al menos un familiar a la intervención (padre o madre).

Los antecedentes son expuestos en la siguiente tabla:

\begin{tabular}{|c|c|c|}
\hline \multicolumn{3}{|c|}{ 2. CASOS } \\
\hline Familia Caso M & Familia Caso S & Familia Caso V \\
\hline $\begin{array}{l}\text { Niña de } 6 \text { años con diagnóstico } \\
\text { de TEA, M. se comunica solo } \\
\text { por gestos y señas y no muestra } \\
\text { lenguaje. En algunas ocasiones } \\
\text { muestra contacto visual. No se } \\
\text { relaciona con los elementos de } \\
\text { juego presentes en la sala. } \\
\text { La familia está compuesta por } \\
\text { madre, padre y hermana mayor } \\
\text { que M. La madre es la cuidado- } \\
\text { ra principal que atiende a M. y } \\
\text { a su hermana, y es quién pasa } \\
\text { mayor tiempo con las niñas } \\
\text { ya que el padre trabaja fuera } \\
\text { de casa. } \\
\text { La madre muestra interés en } \\
\text { la participación del estudio, } \\
\text { argumentando que ella no sabe } \\
\text { cómo jugar con su hija, pues } \\
\text { debido a su rol de cuidadora, } \\
\text { M. solo se relaciona con ella } \\
\text { para satisfacer sus necesidades } \\
\text { básicas. }\end{array}$ & $\begin{array}{l}\text { Niña de } 3 \text { años con diagnóstico } \\
\text { de TEA, } S \text { muestra lenguaje, pero } \\
\text { este no es funcional, ya que } \\
\text { repite lo que escucha de forma } \\
\text { ecolálica. No presenta contacto } \\
\text { visual. No establece juegos. } \\
\text { La familia está compuesta por } \\
\text { madre, padre y S, la madre pasa } \\
\text { la mayor parte del día con S. ya } \\
\text { que el padre trabaja fuera de } \\
\text { casa, quién al regresar de traba- } \\
\text { jar se encarga de los cuidados } \\
\text { de S. } \\
\text { La madre argumenta su interés } \\
\text { en la participación del estudio } \\
\text { para aprender maneras de } \\
\text { comunicarse con S. ya que han } \\
\text { visto avances en su desarrollo, } \\
\text { pero la comunicación sigue } \\
\text { siendo escasa. }\end{array}$ & $\begin{array}{l}\text { V. es un niño de } 4 \text { años, con } \\
\text { diagnóstico de TEA, presenta } \\
\text { lenguaje, pero es pobre y poco } \\
\text { funcional. El contacto visual es } \\
\text { escaso. Se relaciona con los } \\
\text { elementos de juego presentes } \\
\text { en la sala, pero no incluye al } \\
\text { adulto en el mismo. } \\
\text { La familia está compuesta } \\
\text { por su madre, padre y her- } \\
\text { mana mayor de S, siendo } \\
\text { la madre la que pasa mayor } \\
\text { parte del tiempo con } \mathrm{V} \text {. como } \\
\text { cuidadora principal. } \\
\text { El padre argumenta que no } \\
\text { sabe cómo relacionarse con } \mathrm{V} \text {. } \\
\text { ya que la mayoría de las veces } \\
\text { no sabe cómo entender a } \mathrm{V} \text {. } \\
\text { La madre padece una enfer- } \\
\text { medad crónica y en ocasiones } \\
\text { se ve incapacitada por la mis- } \\
\text { ma para el cuidado de } \mathrm{V} \text {, por } \\
\text { lo que necesita el apoyo del } \\
\text { padre con } \mathrm{V} \text {. }\end{array}$ \\
\hline
\end{tabular}


RELACIONES FAMILIARES ENTRE PADRES E HIJOS /AS CON TRASTORNO DE ESPECTRO AUTISTA (TEA) A TRAVÉS DEL JUEGO COMO ACTIVIDAD PRINCIPAL EN LA INTERVENCIÓN DE TERAPIA OCUPACIONAL: ESTUDIO DE CASOS, EN COLEGIOS DE LA COMUNA DE MAIPÚ (RM. CHILE)

\begin{tabular}{|c|c|}
\hline \multicolumn{2}{|c|}{ 3. ENTREVISTA PRE Y POSTINTERVENCIÓN } \\
\hline Preguntas: & Ejemplos orientadores de la pregunta. \\
\hline 1. ¿Cómo juega su hijo? (01) & $\begin{array}{l}\text { (Motivación intrínseca, espontaneidad, disfrute, flexibilidad, } \\
\text { mantenimiento, preferencias en el tipo de juego, elementos u } \\
\text { objetos de juego, metodología organización y rutina de juego, } \\
\text { etc.) }\end{array}$ \\
\hline $\begin{array}{l}\text { 2. ¿Perciben ustedes que el juego es } \\
\text { bueno para su hijo? ¿Me puede deta- } \\
\text { Ilar en qué aspectos? (02) }\end{array}$ & $\begin{array}{l}\text { (Le permite desarrollar habilidades y oportunidades de aprendi- } \\
\text { zaje, le da la oportunidad de desarrollo emocional y autocono- } \\
\text { cimiento, permite exploración libre del ambiente y relacionarse } \\
\text { con los demás, etc.) }\end{array}$ \\
\hline $\begin{array}{l}\text { 3. ¿De qué manera responde su hijo } \\
\text { a sus interacciones dentro y fuera del } \\
\text { juego? (3) }\end{array}$ & $\begin{array}{l}\text { (Las acepta, las permite, las incorpora, atiende, comprende, res- } \\
\text { ponde a ellas, etc.) }\end{array}$ \\
\hline $\begin{array}{l}\text { 4. ¿Cómo se comunica su hijo con } \\
\text { usted? (04) }\end{array}$ & $\begin{array}{l}\text { (Existe contacto visual compartido, indicaciones, gestos, expre- } \\
\text { sión de emociones y respuesta a las mismas, contacto, atención } \\
\text { compartida, imitación, lenguaje intencional, etc.) }\end{array}$ \\
\hline $\begin{array}{l}\text { 5. ¿Cómo se comporta su hijo en lo } \\
\text { social? (05) }\end{array}$ & (Preferencias, intereses, etc.) \\
\hline $\begin{array}{l}\text { 6. ¿Cómo son las relaciones sociales } \\
\text { que establece su hijo con usted? (06) }\end{array}$ & $\begin{array}{l}\text { (Existe inicio espontáneo interés, búsqueda, apoyo, respuesta } \\
\text { emocional, etc.) }\end{array}$ \\
\hline $\begin{array}{l}\text { 7. ¿En qué aspectos contribuye usted al } \\
\text { juego de su hijo? (07) }\end{array}$ & $\begin{array}{l}\text { (Proporciona seguridad, oportunidades, tiempo, libertad de } \\
\text { elección, fomenta la interacción social y el aprendizaje de habi- } \\
\text { lidades y conductas, generalización de aprendizajes, apoyo y } \\
\text { adaptación, etc.) }\end{array}$ \\
\hline $\begin{array}{l}\text { 8. ¿Cómo responde su hijo a los estí- } \\
\text { mulos del ambiente? (08) }\end{array}$ & $\begin{array}{l}\text { (Preferencias, búsquedas sensoriales, reacción a los sonidos, } \\
\text { luces, movimiento, tacto, etc.) }\end{array}$ \\
\hline
\end{tabular}

Se realiza un análisis documental de la bibliografía con el objetivo de recopilar la información publicada relacionada con el tema de investigación. Para ello, se recurrió a la búsqueda en bases de datos (Pubmed, Google Scholar, SCIELO), libros de textos y revistas, de las dimensiones principales del estudio (Juego, familia, interrelaciones y TEA). En base al mismo análisis documental se levantaron categorías y subcategorías de las dimensiones que conforman la Matriz categorial. Posterior a esto, surgieron los reactivos o preguntas que dieron paso a la formación de los instrumentos o herramientas de recolección de datos, tales como: Entrevistas semiestructuradas tanto Pre-intervención y Post-intervención (el cuaderno o Diario de campo y la Pauta de cotejo de las videograbaciones realizadas en las sesiones de intervención, así como el Plan de Intervención orientativo de las sesiones de intervención del estudio.

Las dos primeras sesiones fueron de observación de las relaciones establecidas entre familia y niño con TEA mediante el juego. 
Para recoger la información relevante durante el proceso de intervención se seleccionaron herramientas como el Diario de campo y la video grabación de sesiones.

A partir del Diario de campo, se fueron registrando anotaciones, observaciones del ambiente, descripciones del ambiente o contexto y esquemas para describir dichas relaciones de interacción en el juego.
La videograbación de las sesiones se realizó en dos momentos, uno al inicio de la intervención, otro al final de la intervención, quedando la información recogida en forma de videos, estos fueron analizados por un terapeuta externo mediante una Pauta de cotejo que fue confeccionada y desarrollada a partir de la información recogida en la Matriz categorial de dicho estudio.

\section{PAUTA DE COTEJO: OBSERVACIÓN REGISTRO DE VIDEOS Y COMPARATIVA FINAL CASO S}

\begin{tabular}{|c|c|c|c|c|c|}
\hline \multicolumn{3}{|l|}{ Nombre: $\mathrm{S}$} & Septiembre & Noviembre & \multirow[b]{2}{*}{ Resultado } \\
\hline Dimensión & Categoría & Conducta observada & \multicolumn{2}{|l|}{ Respuesta } & \\
\hline \multirow{12}{*}{ JUEGO } & \multirow{3}{*}{$\begin{array}{l}\text { Motivación } \\
\text { intrínseca }\end{array}$} & $\begin{array}{l}\text { ¿El niño elige un juego determinado por } \\
\text { motivación propia? }\end{array}$ & si & si & - \\
\hline & & $\begin{array}{c}\text { ¿El niño inicia el juego de manera } \\
\text { espontánea? }\end{array}$ & A veces & si & M \\
\hline & & $\begin{array}{c}\text { ¿Disfruta del juego? (Se ríe, lo repite, } \\
\text { pide más, etc.) }\end{array}$ & si & si & - \\
\hline & \multirow{3}{*}{ Desarrollo } & $\begin{array}{c}\text { ¿Muestra algún tipo de aprendizaje } \\
\text { durante el juego? }\end{array}$ & si & si & - \\
\hline & & $\begin{array}{c}\text { ¿El juego le permite experimentar y } \\
\text { descubrir cosas nuevas? }\end{array}$ & A veces & si & M \\
\hline & & $\begin{array}{c}\text { ¿Le permite explorar sus capacidades y } \\
\text { límites? }\end{array}$ & no & no & - \\
\hline & \multirow{6}{*}{ Tipo de juego } & $\begin{array}{c}\text { ¿Mantiene el juego en un espacio } \\
\text { determinado de tiempo? }\end{array}$ & si & si & - \\
\hline & & ¿Se muestra involucrado en el juego? & si & si & - \\
\hline & & $\begin{array}{c}\text { ¿Muestra preferencias en cuanto a } \\
\text { un juguete o un elemento de juego } \\
\text { determinado? }\end{array}$ & si & si & - \\
\hline & & ¿Presenta juego solitario? & si & no & $\mathrm{S}$ \\
\hline & & ¿Comparte el juego? & A veces & si & M \\
\hline & & ¿Muestra juego simbólico? & no & no & - \\
\hline
\end{tabular}




\begin{tabular}{|c|c|c|c|c|c|}
\hline Dimensión & Categoría & Conducta observada & \multicolumn{2}{|c|}{ Respuesta } & Resultado \\
\hline \multirow{15}{*}{$\begin{array}{l}\text { INTERRELA- } \\
\text { CIONES }\end{array}$} & \multirow{4}{*}{$\begin{array}{l}\text { Comunica- } \\
\text { ción verbal }\end{array}$} & ¿Muestra lenguaje funcional? & no & A veces & M \\
\hline & & ¿Atiende y comprende lenguaje verbal? & no & A veces & M \\
\hline & & ¿Responde al lenguaje verbal? & A veces & A veces & - \\
\hline & & $\begin{array}{l}\text { ¿Hay copia e imitación de lo que el } \\
\text { adulto dice? }\end{array}$ & no & si & S \\
\hline & \multirow{7}{*}{$\begin{array}{l}\text { Comunica- } \\
\text { ción no verbal }\end{array}$} & ¿Muestra contacto visual? & A veces & si & M \\
\hline & & $\begin{array}{c}\text { ¿Se mantiene el contacto visual en el } \\
\text { tiempo? }\end{array}$ & no & si & S \\
\hline & & ¿Hace uso instrumental del adulto? & si & A veces & M \\
\hline & & ¿Utiliza gestos para comunicarse? & no & no & - \\
\hline & & $\begin{array}{l}\text { ¿Muestra emociones mediante expre- } \\
\text { siones faciales? }\end{array}$ & A veces & no & - \\
\hline & & $\begin{array}{c}\text { ¿Hay imitación o copia de gestos del } \\
\text { adulto? }\end{array}$ & no & A veces & M \\
\hline & & $\begin{array}{c}\text { ¿Hay atención compartida con el } \\
\text { adulto? }\end{array}$ & A veces & si & M \\
\hline & \multirow{4}{*}{$\begin{array}{l}\text { Iniciativa en } \\
\text { las Interrela- } \\
\text { ciones }\end{array}$} & $\begin{array}{c}\text { ¿Busca el niño interacción con el } \\
\text { adulto? }\end{array}$ & A veces & si & M \\
\hline & & $\begin{array}{c}\text { ¿Inicia el niño la interacción con el } \\
\text { adulto? }\end{array}$ & A veces & si & M \\
\hline & & 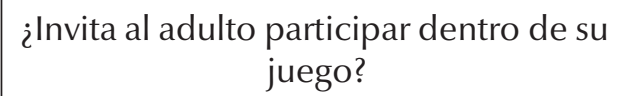 & A veces & A veces & - \\
\hline & & $\begin{array}{l}\text { ¿Permite que el adulto sea participe de } \\
\text { su juego? }\end{array}$ & si & si & - \\
\hline \multirow{4}{*}{ FAMILIA } & & $\begin{array}{l}\text { ¿Permite la familia la elección de juego } \\
\text { libre en el niño? }\end{array}$ & A veces & si & M \\
\hline & & ¿Respeta el juego elegido por el niño? & si & si & - \\
\hline & & ¿Sigue el juego del niño? & si & si & - \\
\hline & & $\begin{array}{l}\text { ¿La familia premia los aciertos o retos } \\
\text { conseguidos dentro del juego? }\end{array}$ & si & si & - \\
\hline \multirow[b]{2}{*}{ SOCIAL-TEA } & \multirow{2}{*}{$\begin{array}{l}\text { Respuesta a } \\
\text { los estímulos } \\
\text { sensoriales }\end{array}$} & $\begin{array}{c}\text { ¿Hay algún tipo de búsqueda sensorial } \\
\text { en el niño? }\end{array}$ & si & si & - \\
\hline & & $\begin{array}{c}\text { ¿Busca un juguete o tipo de juego que } \\
\text { le proporciona la experiencia sensorial } \\
\text { que necesita? }\end{array}$ & si & si & - \\
\hline
\end{tabular}


Los resultados fueron obtenidos mediante: Entrevista pre y post intervención, Diario de campo y Videograbación de las sesiones de intervención. Posterior a su recolección, la Entrevista pre-intervención y Entrevista post- intervención y el Diario de campo fueron transcritos, marcados y codificados para su posterior análisis descriptivo.

La Pauta de cotejo de videos se utilizó para comparar la información audiovisual de cada caso, al principio y tras el proceso de intervención: Se estableció un cuadro comparativo donde se observaba la aparición, modificación o desaparición de las conductas y/o comportamientos analizados tanto de la familia como de los niños en base a las categorías y subcategorías preestablecidas desde la Matriz categorial de cada caso y se relevó como hallazgos las categorías en las que se encontraron modificaciones tras el proceso de intervención presentes en los tres casos.

Finalmente, la información del análisis de las Entrevistas (pre-intervención y post-intervención) fue comparada entre sí, y a su vez se comparó con la información del análisis descriptivo del Cuaderno de campo y con el resultado de la información de la Pauta de cotejo de vídeos. Respondiendo a los supuestos iniciales de sí las interacciones sociales entre familia e hijo con TEA, eran SUSTANTIVAMENTE MODIFICADAS, MEDIANAMENTE MODIFICADAS o NO eran MODIFICADAS por la intervención de la familia en el tratamiento de Terapia Ocupacional en el juego.

Encontrándose finalmente como hallazgos, la modificación MEDIANA de conductas observadas respecto a al juego; tales como el aumento de la motivación intrínseca de juego en los niños y la importancia del juego como medio y ocupación de aprendizaje para el desarrollo del niño.

\section{Resultados}

Se observaron, además, tras el proceso de intervención, modificaciones SUSTANTIVAS en el aumento del juego compartido entre el niño y la familia pasando de un tipo de juego solitario observado en el niño al inicio de esta, a un juego compartido con la familia.

En cuanto a la categoría establecida según la Matriz Categorial como Desarrollo de juego: La familia encuentra que hay mayor interacción social en el desarrollo de juego, hay mayor aceptación del adulto, en muchas ocasiones el niño busca al adulto para compartir su juego con él y también han aumentado la búsqueda de interacción con sus iguales.

Hay mayor flexibilidad y desarrollo de diferentes tipos de juego. Y mayor inicio de juego espontáneo por su parte.

De las anotaciones del Cuaderno de Campo durante las sesiones Pre-intervención se recoge que para el Caso $\mathrm{V}$ «Juega solo y de manera repetitiva», lo que podemos comparar con la información recogida en la entrevista Post-intervención donde la familia señala: «ahora juega con papá, eh, con la hermana también tiene otro tipo de juego, entonces yo creo que igual ha sido bueno porque ha incorporado más personas.»

Con respecto a los cambios observados en las relaciones establecidas entre familia y niño se obtiene como resultado una modificación MEDIANA. Como, por ejemplo, en la comunicación verbal establecida (donde se observa el aumento del lenguaje verbal en 2 de los 3 niños), mayor atención y comprensión del lenguaje y mayor copia e imitación del lenguaje del adulto, considerándose este aspecto como SUSTANTIVAMENTE modificado en uno de los tres casos.

Para la categoría establecida como Interacción: Para el Caso $\mathrm{S}$ se recoge de las anotaciones del cuaderno de Campo lo siguiente: «No presenta contacto visualy hace uso instrumental del adulto. Sin funcionalidad comunicativa en su lenguaje».

Tras la intervención, recogemos de la entrevista Postintervención realizada a la familia:

«sí se daba cuenta que en su entorno había cosas entretenidas y que los niños también estaban como jugando también igual que ella, y eso ha cambiado porque ella no, ni en la sala cuna ni en ningún lugar había interactuado, así como con niño, o sea, incluso cuando era más más chiquitita se le acercaba un niño y ella como que así lo apartaba y hasta le pegó a una niña, como que no tenía intención de mirar siquiera a un niño.»

Las familias observan que los niños se muestran más conscientes de la presencia de otros niños, comparten 
mayor interacción con sus iguales y mayores (miradas, gestos, expresiones, contacto, imitación y copia)

En la comunicación no verbal, se observa una modificación MEDIANA en las relaciones establecida entre familia e hijo. Destacándose la modificación de aspectos como el aumento de la atención compartida o conjunta con el adulto y el uso del lenguaje no verbal: el niño utiliza mayores gestos para comunicarse con la familia.

Dentro de la categoría establecida como Comunicación, para el caso $M$ se recoge de la entrevista Preintervención a la familia: «Son los pocos momentos en los que tienes una interacción directa, y donde no hay una necesidad.»

Información que podemos comparar con lo recogido en la entrevista Post-intervención a la familia:

«Ahora no, tenemos que darnos el tiempo de saber qué es lo que quiere porque ella intenta comunicarse, intenta comunicarse más, cachai, no verbalmente, pero sí con [con] los gestos que ella hace, entonces ahora hay que darse el tiempo de ver qué es lo que quiere.

Se observa en los niños mayor intención comunicativa (Hacen mayor uso de gestos y lenguaje para comunicar lo que quieren).

También se observa el aumento del contacto visual del niño, como SUSTANTIVAMENTE modificado, así como el mantenimiento de este en las relaciones establecidas.

Para el caso S dentro de esta misma categoría de comunicación se recoge de la Entrevista Post-intervencion:

Sí, mira, en un principio era solo a mí y a Maxi, ahora como que está mirando más a las personas en sí cuando, eh, como que cuando quiere expresar algo como que está tomando el contacto visual con la, con las demás personas igual. (E2S)

El contacto visual ha aumentado notablemente y se hace con más personas fuera del ambiente familiar.

Además, los padres señalan que se muestra un aumento en intención comunicativa del niño, así como en la comprensión y expresión de emociones por su parte.
En la interacción entre familia y niño hay una modificación MEDIANA con respecto a la participación de juego por parte adulto, el niño busca al adulto, lo invita a participar en el mismo, y lo comparte las experiencias de juego con él.

Como muestra de ello dentro de la categoría de Familia se recoge de la entrevista Post-intervención del caso $M$ :

Ahora sí, ahora que yo entendí, comprendí y acepté que esos son los juegos de mi hija, sí, ahora sípo, yo juego con ella a lo que quiere jugar ella, no a lo que quiero jugar yo, ni a lo que quiere jugar el papá, ni a lo que quiere jugar la hermana, eso es lo que quiere jugar la hermana, o sea, la M: sí, entonces sí hubo como un cambio, sí, harto, harto, harto; es comprender no más po.

Conforme van desarrollándose las intervenciones y tras acompañar al niño de las experiencias sensoriales que busca y disfruta, los momentos de interacción dentro del juego van desarrollándose, apareciendo mayor contacto visual, atención compartida, imitación y copia, interacción social, permanencia de juego, y disfrute.

La intervención ayuda a los padres a comprender, aceptar y compartir la etapa de desarrollo de juego en la que está su hijo, así como a respetar y a compartir la elección de juego del niño y a partir de ahí las interacciones de juego se han visto modificadas.

\section{Discusıón}

Los resultados obtenidos refuerzan las teorías que dan base al estudio como la Teoría General de Sistemas (Ludwig von Bertalanffy, 1968) o el Modelo Ecológico de Bronfenbrenner (Gifre \& Guitart, 2013), las cuales sitúan a la familia como sistema abierto y cambiante.

A raíz de los resultados del estudio se puede observar que las relaciones de interacción dentro de la familia pueden ser modificadas desde el interior de esta, y así a su vez generar cambios observables en las conductas tanto de padres como de hijos con TEA.

Por consiguiente, situando a la familia como entorno primario del niño y modificando las interacciones sociales que establece esta con su hijo con TEA dentro del ambiente de juego, las relaciones sociales establecidas entre el niño y su medio también se ven modificadas. 
En oposición a los estudios ya referenciados con anterioridad (Vernon, Koegel, Dauterman, \& Stolen, 2012; Beaudoin, Sébire, \& Couture, 2014; McKnight, O'Malley-Keighran, \& Carroll, 2016; Zlomke, Jeter, \& Murphy, 2017), desde este estudio, se observa un cambio en el foco de la investigación, ya que no se pretende establecer un programa educativo hacia los padres, sino explorar y reconocer las relaciones entre familia y niño con TEA, los cambios y modificaciones en las relaciones establecidas entre ambos, mediante el juego como actividad principal en la intervención de Terapia Ocupacional.

Se contempla que el aprendizaje de los padres debe ser en base a sus propias experiencias y observación del niño en su interacción dentro el contexto de juego, que no ha de surgir meramente de las pautas o recomendaciones que el Terapeuta Ocupacional pueda dar, sino de las propias dinámicas de interacción que surgen entre la familia y el niño y de la manera de relacionarse del propio niño dentro de un ambiente de juego, que si bien no es del todo espontáneo dado que se desarrolla dentro de un contexto de intervención, intenta replicar las características del mismo en cuanto a motivación intrínseca y exploración libre del ambiente.

Se puede agregar que además se observaron modificaciones en la interacción social establecida entre el niño con TEA y su familia (tales como aumento del contacto visual, atención conjunta, intención comunicativa, lenguaje expresivo y comprensivo, etc.). Cambios considerables, teniendo en cuenta las características diagnósticas del TEA que afectan fundamentalmente a la interacción social (American Psychiatric Association, 2014).

Además, se encontró que, mediante las interacciones establecidas dentro del ambiente de juego, las relaciones establecidas entre familia y niño fueron aumentando, surgiendo nuevas conductas de interacción en los niños (mayor intención comunicativa, mayor contacto visual, mayor uso del lenguaje tanto comprensivo como expresivo, mayor comprensión y expresión de emociones por parte del niño) y a la vez, fruto de la misma interacción de juego, los padres aprendían más herramientas para relacionarse con sus hijos; lo que refuerza la información planteada por Vernon, Koegel, Dauterman, \& Stolen (2012) que encontraron aumentos en todas las áreas del funcionamiento social medidas, incluido el contacto visual con los niños, las iniciaciones verbales y el afecto positivo dirigido y simultáneamente, además de un aumento en el afecto positivo de los padres y el compromiso sincrónico. (Vernon, Koegel, Dauterman, \& Stolen, 2012)

Con respecto a la importancia del juego, la familia encuentra que hay mayor interacción social en el desarrollo de juego del niño, mayor aceptación del familiar como compañero de juego, siendo el niño el que inicia la búsqueda del adulto para compartir su juego con él. Y, además, la familia observa que aumentó la búsqueda de interacción con iguales en el ambiente de juego. Estos resultados, refuerzan la información de otros estudios que dan cuenta de la importancia de la interacción durante el contexto de juego para el desarrollo social del niño, "si los padres estuvieran involucrados durante este proceso de intervención, uno podría predecir poderosos efectos colaterales sobre su participación" (Vernon, Koegel, Dauterman \& Stolen, 2012), debido a que los efectos de la presencia de los padres en la intervención y en la participación del juego con sus hijos aumenta la interacción social tanto en el contexto de juego como en otros contextos, extendiéndose más allá de este.

Datos de estudios anteriores (Agazzi, Tan, Ogg, Armstrong \& Kirby, 2017) fueron corroborados en este estudio tras los resultados que declara la familia sobre el aumento de seguridad en la crianza de sus hijos. Ya que a medida que se establece una mayor interacción entre familia y niño mediante el juego, la familia adquiere un mayor conocimiento de la manera en la que establecer relaciones con su hijo, y esto le proporciona un mayor conocimiento del niño y un mayor empoderamiento en cuanto a las funciones parentales sobre el cuidado de este.

Si bien Kasari y otros (2012), encontraron como resultado de su estudio una generalización del aumento de la atención conjunta y juego en el juego compartido con sus madres en este estudio se da muestra de que el hecho de que la familia forme parte del proceso de interacción permite un mayor aprendizaje y desarrollo de habilidades para relacionarse con sus hijos en base a las propias características del niño. Y que este aprendizaje, puede ser compartido con otras personas importantes del entorno del niño (familia, entorno cercano, demás profesionales que trabajan con el niño, etc.).

Zlomke, Jeter \& Murphy (2017) encontraron altos niveles de aceptabilidad de los padres hacia la intervención 
coincidiendo con las consideraciones sobre la familia en este estudio respecto a la apreciación del tipo de intervención realizada, ya que la familia considera como muy valorable los conocimientos adquiridos durante la intervención, debido a que han podido comprender mucho mejor al niño en las relaciones establecidas entre ambos, y a su vez, han podido transmitir este aprendizaje tanto a otros miembros de la familia como a otros profesionales que trabajan con el niño.

De los hallazgos o aportes más relevantes que emergen del propio estudio cabe resaltar las características de la propia intervención desde Terapia Ocupacional, la cual invita a los padres a participar desde el interior de la intervención, haciéndolos formar parte del proceso de tratamiento de su hijo, estableciendo modificaciones en las relaciones que mantienen entre ambos gracias al juego. Esto aporta a los padres más información y conocimiento sobre sus hijos. Ellos manifiestan que gracias a las experiencias de juego aprenden una gama mayor de herramientas para interactuar con sus hijos, y es lo que la experimentación de la observación y el compartir las experiencias de juego junto a su hijo les trasmite.

Solo haciendo partícipes a la familia de las experiencias de juego como actividad y herramienta fundamental para el Terapeuta Ocupacional in situ, pueden comprender, experimentar y modificar aspectos relacionales con sus hijos. Destacando aún más si cabe, el hecho de la afectación al establecimiento de relaciones sociales que presentan sus hijos debido al diagnóstico de TEA.

Debido también a la relevancia del juego como actividad dentro del tipo de intervención establecida, los padres han alcanzado a comprender la importancia y significado que tiene esta actividad para el desarrollo de sus hijos, emergiendo una organización de sus horarios y rutinas para destinar un espacio de tiempo dedicado a compartir esta actividad con sus hijos en su día a día.

Concluyendo que las relaciones de interacción entre padres e hijos con TEA pueden verse modificadas mediante el juego como actividad y ocupación principal y relevante para el desarrollo del niño. Encontrando resultados positivos en conductas novedosas de interacción tanto para las familias como para los niños.

Los hallazgos de esta investigación actual, aunque son prometedores, deben ser atenuados por las limitaciones del tamaño de la muestra del estudio y las dificultades de igualar las condiciones y características de las familias del estudio.

Cabe destacar como limitación la experticia del Terapeuta Ocupacional en el conocimiento y experiencia en intervención con TEA y familia y los distintos modelos teóricos y estrategias de intervención desde los que se fundamenta la base de dicha intervención de Terapia Ocupacional dentro de este estudio.

Para ello, con este estudio se abren futuras líneas de investigación con el objetivo de seguir explorando en la temática sobre la modificación de las relaciones con una mayor muestra de población, y poder cuantificar dichas modificaciones para poder generalizar los resultados y determinar en mayor grado la efectividad de las modificaciones de las conductas novedosas dentro de las relaciones establecidas entre padres e hijos con TEA tras la intervención del juego.

No hubo fuente de financiamiento para dicho estudio.

\section{RefERENCIAS BibLIOGRÁFICAS}

Agazzi, H., Tan, S. Y., Ogg, J., Armstrong, K., \& Kirby, R. S. (2017). Does Parent-Child Interaction Therapy Reduce Maternal Stress, Anxiety, and Depression Among Mothers of Children with Autism Spectrum Disorder? Child \& Family Behavior Therapy, 39(4), 283- 303. https://doi.org/10.1080/07317107.2017.1375622

American Psychiatric Association. (2014). DSM-5. Manual Diagnóstico y Estadístico de los Trastornos Mental.

Beaudoin, A. J., Sébire, G., \& Couture, M. (2014). Parent training interventions for toddlers with autism spectrum disorder. Autism Research and Treatment, 2014, 839-890. https://doi. org/10.1155/2014/839890

Brezis, R., Weisner, T., Daley, T., Singhal, N., Barua, M., \& Chollera, S. (2015). Parenting a Child with Autism in India: Narratives Before and After a Parent-Child Intervention Program. Culture, Medicine \& Psychiatry, 39(2), 277-298. https://doi.org/10.1007/ s11013-015-9434-y

Dankhe G. L. (1976). Investigación y comunicación, en C. Fernández-Collado y G.L., (La comunicación humana: ciencia social). México D.F.

Departamento de Discapacidad y Rehabilitación, División de Prevención y Control de Enfermedades, Subsecretaría de salud pública, \& Ministerio de Salud de Chile. (2011). Guía de Práctica Clínica: Detección y Diagnóstico Oportuno de los Trastornos del Espectro Autista. 
Gifre, M., \& Guitart, M. E. (2013). Consideraciones educativas de la perspectiva ecológica de Urie Bronferbrenner. Contextos Educativos. Revista de Educación, (15), 79-92. https://doi.org/10.18172/con.656

Hernández Sampieri, C. R. H., Collado, C. F., \& Lucio, P. B. (2010). Metodología de la investigación. McGraw-Hill Interamericana de México, S.A. de C.V.

Kasari, C., Gulsrud, A., Freeman, S., Paparella, T., \& Hellemann, G. (2012). Longitudinal Follow Up of Children with Autism Receiving Targeted Interventions on Joint Attention and Play RH = Targeted Interventions on Joint Attention and Play. Journal of the American Academy ofChild and Adolescent Psychiatry, 57(5), 487-495. https:// doi.org/10.1016/j.jaac.2012.02.019

López, \& Polonio. (2014). Terapia Ocupacional en la Infancia. Médica Panamericana.

Ludwig von Bertalanffy. (1968). Teoría General de los Sistemas. Recuperado de http://www.academia.edu/download/38846231/teoria.pdf

McConachie, H., \& Diggle, T. (2007). Parent implemented early intervention for young children with autism spectrum disorder: a systematic review. Journal of Evaluation in Clinical Practice, 13(1), 120-129. https://doi.org/10.1111/j.1365-2753.2006.00674.x

McKnight, L. M., O'Malley-Keighran, M.-P., \& Carroll, C. (2016). 'Just wait then and see what he does': a speech act analysis of healthcare professionals' interaction coaching with parents of children with autism spectrum disorders. International Journal of Language \& Communication Disorders, 57(6), 757-768. https:// doi.org/10.1111/1460-6984.12246

Parham, L. D., \& Fazio, L. (2007). Play in a occupational therapy for children. Mosby.

Schultz, T. R., Stichter, J. P., Herzog, M. J., McGhee, S. D., \& Lierheimer, K. (2012). Social Competence Intervention for Parents (SCI-P): Comparing Outcomes for a Parent Education Program Targeting Adolescents with ASD. Autism Research and Treatment. https:// doi.org/10.1155/2012/681465

Vernon, T. W., Koegel, R. L., Dauterman, H., \& Stolen, K. (2012). An Early Social Engagement Intervention for Young Children with Autism and their Parents. Journal of autism and developmental disorders, 42(12), 2702-2717. https://doi.org/10.1007/s10803-012-1535-7

Zlomke, K. R., Jeter, K., \& Murphy, J. (2017). Open-Trial Pilot of ParentChild Interaction Therapy for Children with Autism Spectrum Disorder. Child \& Family Behavior Therapy, 39(1), 1-18. https:// doi.org/10.1080/07317107.2016.1267999 